\title{
Cold Electrostatic Ion Cyclotron Waves for Preionization and IBW Launching in LHD*
}

\begin{abstract}
Masayuki Ono
Princeton Plasma Physics Laboratory, Princeton University, New Jersey, USA.

ABSTRACT. A folded waveguide with $\mathrm{E}_{\|}$polarization is being installed on LHD device. The main purpose of the folded waveguide is to pre-ionize and create good target plasmas. The present manuscript proposes a launching of IBW via CESICW (Cold Electrostatic Ion Cyclotron Wave) for heating the core of LHD with the folded waveguide. The core heating can be accomplished by adding a minority hydrogen ion species in a helium majority plasma facilitating the mode-transformation of CESICW into IBW at the ion-ion hybrid resonance.
\end{abstract}

\section{INTRODUCTION}

Due to the absence of strong ohmic heating, a non-ohmic plasma production is an essential part of the stellarator research. Electron cyclotron heating $(\mathrm{ECH})$ is a well-known effective tool for plasma production and electron heating. Possible limitations for $\mathrm{ECH}$ are the fixed magnetic field requirement (due to a fix frequency nature of a given ECH source) and the density limit. A folded waveguide is installed on LHD to develop an alternate plasma production method in the ion cyclotron range of frequency. ${ }^{1}$ The transmitters in this frequency range can be made to be tunable for a range of frequency giving some magnetic field operational flexibility. The plasma production in this frequency range therefore has a promise of giving economical and flexible approach to the stellarator plasma production. This manuscript explores a possibility of core plasma heating with the folded waveguide on LHD.

\section{CESICW LAUNCHING AND ACCESSIBILITY}

One possible mode to use for the $\mathrm{E}_{\mid}$polarized folded waveguide is the cold electrostatic ion cyclotron wave (CESICW). ${ }^{2}$ Excitation and absorption of this mode has been previously investigated using a phased electrostatic launcher in the Princeton L-4 and ACT-1 devices. ${ }^{2}$ Since this wave is electrostatic and strongly Landau damped, it can be used to heat electrons efficiently. The cold plasma electrostatic wave dispersion relation for CESICW can be written as

$$
S n_{\perp}^{2}+P\left(n_{\|}^{2}-S\right)=0
$$

where $S=\left(1+\frac{\omega_{p H}^{2}}{\left(\Omega_{H}^{2}-\omega^{2}\right)}-\frac{\omega_{p H e}^{2}}{\left(\omega^{2}-\Omega_{H e}^{2}\right)}\right)$ and $P=\left(1-\frac{\omega_{p e}^{2}}{\omega^{2}}\right) . \quad$ For the purpose of the LHD application, the ion species assumed in this paper are hydrogen and helium. As can be seen from Eq. 1, for a given $n_{||}^{2}$, CESICW exhibits a perpendicular cut-off $\left(\lambda_{\perp} \Rightarrow \infty\right)$ at the ion cyclotron frequency $\Omega_{i}$ and a resonance $\left(\lambda_{\perp} \Rightarrow 0\right)$ at the ion-ion hybrid resonance frequency $\omega_{\mathrm{ii}}$. In other 
words, CESICW exists between $\Omega_{H}$ and $\omega_{\mathrm{ii}}$. In order to launch the wave, it is important to consider the wave group velocities and wavelengths. In general, it is desirable to launch plasma waves with high perpendicular group velocity $v_{g \perp}$ and relatively large perpendicular wavelength $\lambda_{\perp}$ The CESICW $v_{g \perp}$ goes to zero as $\omega \Rightarrow \Omega_{i}$ and also as $\omega \Rightarrow \omega_{i i}$. So, an optimum launching point lies somewhere in between these two limiting frequencies. Another point to note is that the wave propagation angle tends to be steeper (or $v_{g \perp} / v_{g \|}$ increases) as $\omega \Rightarrow \Omega_{i}$. Therefore, an optimum launching frequency is somewhat below $\Omega_{H}$. It is also informative to examine the wave dispersion relation from the vacuum into plasma interior. At the electron plasma frequency $P=0$, the wave starts to propagate as an electron plasma wave (EPW). For $25 \mathrm{MHz}$ frequency, this corresponds to the plasma density of only $8 \times 10^{6} \mathrm{~cm}^{-3}$ which can be easily exceeded at the launcher surface. The EPW then changes into a CESICW near the ion plasma frequency which is $n_{e} \approx 10^{10} \mathrm{~cm}^{-3}$ plasma density. Therefore a continuous electrostatic wave propagation is possible from the launcher surface to CESICW for this frequency range.

\section{CESICW - ICW MODE-CONVERSION}

As CESICW propagates into the plasma interior, it encounters a "cutoff" at the Alfven resonance, $n_{||}^{2}=S$, as shown in Eq. (1). Actually, CESICW modeconverts into the (electromagnetic) Ion Cyclotron Wave (ICW) at the Alfven resonance preventing further wave penetration. This physics of the modeconversion can be described well by the following quadratic cold plasma wave equation in $n_{\perp}^{2}$ for a given $n_{||}^{2} \cdot 3$

$$
a n_{\perp}^{4}+b n_{\perp}^{2}+c=0
$$

where $a=S, \quad b \cong P\left(n_{||}^{2}-S\right)$ and $c=P\left[\left(n_{||}^{2}-S\right)^{2}-D^{2}\right]$. There are two limiting solutions of Eq. (2). The shorter wavelength solution is $n_{\perp}^{2}=-b / a$ which is CESICW, and the longer wavelength solution is $n_{\perp}^{2}=-c / b$ which is an outgoing ICW. The mode conversion takes place when $b^{2}=4 a c$ which is near the Alfven resonance, $n_{||}^{2}=S$. A schematic for CESICW-ICW Mode Conversion is shown in Fig. 1. Since the WKB approximation breaks down near the modeconversion point, there is also a possibility of CESICW reflection back at the mode-conversion point. At any rate, CESICW can only penetrate up to the modeconversion point which can range from $n_{e}=10^{10}-10^{12} \mathrm{~cm}^{-3}$ depending on the value of $n_{||}^{2}$. It should be noted that the accessibility problem exists even if ICW is directly excited here since ICW also mode-converts into CESICW at the Alfven resonance. 


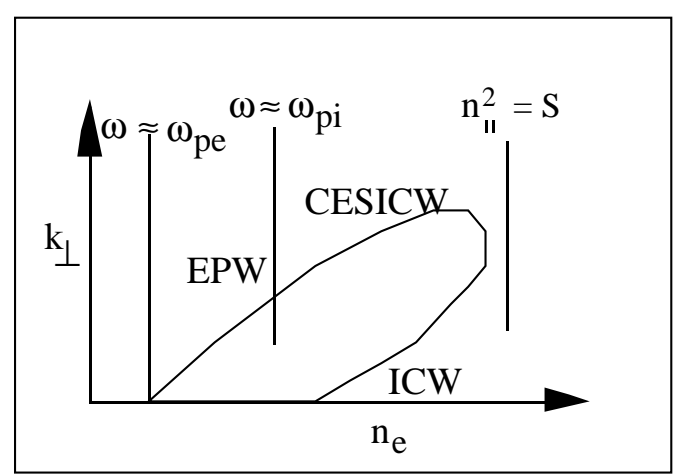

Fig. 1, CESICW-ICW

Mode-Conversion Process

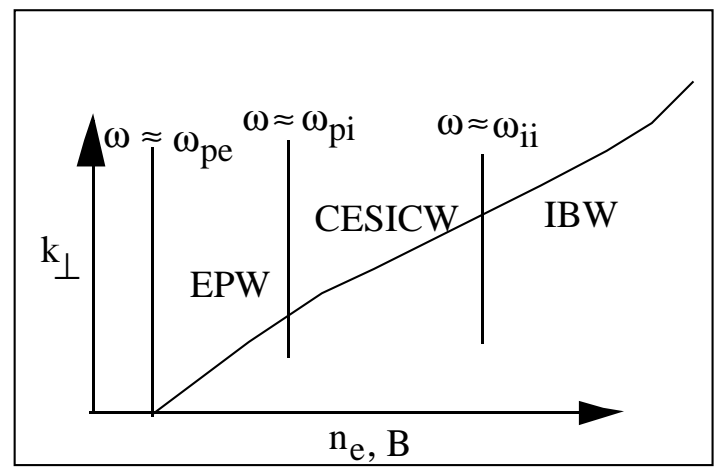

Fig. 2. CESICW-IBW

Mode-Transformation Process

\section{CESICW - IBW MODE-TRANSFORMATION}

It is possible to improve the wave accessibility into higher density if CESICW can mode-transform into Ion Bernstein Wave (IBW) which has no density limit. ${ }^{4}$ Due to the finite-Larmor-radius effect, CESICW mode-transforms into IBW at the ion-ion hybrid resonance layer or $\omega=\omega_{i i}$, provided that CESICW does not suffer a mode-conversion into ICW before reaching the $\omega=\omega_{i i}$ "resonance". This possibility has been explored previously for the TFTR IBW experiment. ${ }^{5}$ The CESICW-IBW mode-transformation can be described by the following quadratic equation in $n_{\perp}^{2}$ by retaining the first order ion-Larmor-radius terms in $S$.

$$
A k_{\perp}^{4}+B k_{\perp}^{2}+C=0
$$

where $A=3 \omega_{p H e}^{2}\left(T_{H e} / m_{H e}\right) /\left[\left(4 \Omega_{H e}^{2}-\omega^{2}\right)\left(\omega^{2}-\Omega_{H e}^{2}\right)\right]$, , $B=\omega_{p H}^{2} /\left(\Omega_{H}^{2}-\omega^{2}\right)-\omega_{p H e}^{2} /\left(\omega^{2}-\Omega_{H e}^{2}\right)$, and $C=-\left(\omega_{p e}^{2} / \omega^{2}\right) k_{||}^{2}$. Here the longer wave solution $k_{\perp}^{2}=-C / B$ is CESICW and the shorter wave solution $k_{\perp}^{2}=-B / A$ is IBW. A schematic of this process is shown in Fig. 2. The finite ion-Larmor-radius effect eliminates the CESICW resonance at $\omega=\omega_{i i}$ or $b=0$. From Eq. 3, one can see that the wave solution does exist as $k_{\perp}^{2}=\sqrt{-C / A}$ even at $\omega=\omega_{i i}$. The WKB solution is usually satisfied through the CESICW-IBW mode-transformation process that the conversion into IBW should be quite efficient. The physics of CESICW - IBW mode-transformation has also been previously validated by a full wave kinetic slab code, SEMAL. 6 The strategy therefore is to launch CESICW in a helium plasma with a minority hydrogen species. This creates $\omega=\omega_{i i}$ layer relatively close to $\omega=\Omega_{H}$. The wave launching window is the region between $\omega=\omega_{i i}$ and $\omega=\Omega_{H}$ layers which width can be adjusted by the minority hydrogen concentration. 


\section{LHD FOLDED WAVEGUIDE APPLICATION}

For the LHD application, it is important to consider the experimental conditions carefully. The LHD folded waveguide is placed in the low field side of the plasma. While this location is favorable from the CESICW and IBW launching point of view, this region is also a divertor $\mathrm{x}$-point region where large heat and particle flows are expected to impinge on the launcher. This region is not suitable to propagate the finite ion temperature IBW mode since the plasma temperature is expected to be low and the density to be high. Instead, CESICW which is a cold electrostatic mode should be utilized to propagate through the divertor region into the main plasma. As noted above, CESICW will have a limited plasma access due to the mode-conversion into ICW. As the launched CESICW propagates away from the launcher, it experiences increased magnetic field along the field line. This causes down shifting of wave $n_{\mid !}^{2}$. This down shifting of $n_{\mid}^{2}$ will accelerate the mode-conversion process into ICW. This effect preclude core heating and would also likely to result in an ineffective plasma production. For more effective plasma production and heating, it is important to deliver the wave power into the plasma core. It is therefore important for CESICW to mode-transform into IBW as described above. Once the mode-transformation into IBW takes place, IBW should propagate into the plasma core along the trough of the magnetic field. The wave absorption will be mainly by electron Landau damping. In this relatively low frequency regime, it may be possible to heat impurity ions with IBW as the IBW encounters the impurity cyclotron harmonic resonances. One can also add minority $\mathrm{He}^{3}$ which could also provide an effective ion absorption layer.

\section{CONCLUSIONS AND DISCUSSIONS}

In summary, a CESICW-IBW mode-transformation scenario in a heliummajority/hydrogen-minority plasma offers a promising possibility for plasma production and core heating utilizing the $\mathrm{E}_{\|}$polarized folded waveguide in LHD. For core heating, it is important for CESICW to mode-transform into IBW before reaching the Alfven resonance. It is also important to launch CESICW with sufficiently high $n^{2}$ to help the core electron heating by IBW. Some ion heating may be possible through residual impurity ions and/or through addition of minority ions such as $\mathrm{He}^{3}$. It is important to perform 3-D ray-tracing calculations in a realistic LHD geometry to assess the actual wave trajectories and power deposition profiles.

*This work was supported by US. D.o.E. Contract DE-AC02-76CH03073. The author thanks helpful discussions with Drs. T. Watari and R. Kumazawa.

\section{REFERENCES}

1 R. Kumazawa et al., at this conference.

2 M. Ono, Phys. Rev. Letters 42, 1267 (1979)

3 T.H. Stix, Waves in Plasmas, AIP (1992).

4 M. Ono, Phys. Fluids B5, 241 (1993).

5 M. Ono et al., AIP Conference Proceedings 403 (New York, 1997) 301.

6 O. Sauter and J. Vaclavik, Computer Physics Comm. 84, 226 (1994). . 